\title{
MASS SPECTROMETRY IN DRUG DISCOVERY AND DEVELOPMENT
}

Broadly trained
scientists with
mass spectro-
metry technical
skills add great
value to drug
discovery teams.

John Michnowicz is Proteomics Program Manager, Life Sciences and Chemical Analysis, Agilent Technologies, 1601 California Avenue, Palo Alto, California 94304, USA. e-mail:

john_michnowicz@agilent.com
During the past decade, the technological improvements in mass spectrometry (MS) for the analysis of small-molecule drugs and biomolecules have had a significant impact on drug discovery and development. Modern LC/MS (liquid chromatography/mass spectroscopy) using the various forms of atmospheric-pressure ionization (API) and matrix-assisted laser desorption ionization (MALDI) have made the identification and quantification of complex and liable molecules a routine procedure. The ability to analyse low levels of oligonucleotides, peptides, proteins and small-molecule drugs in automated, high-throughput assays has been greatly enhanced by the capabilities of modern MS. Examples of areas that have been made more routine by the use of modern MS are the mapping of singlenucleotide polymorphisms, protein-target identification, the confirmation of combinatorial-chemistry synthesis, adsorption, distribution, metabolism, excretion (ADME) assays and traditional small-molecule quality assurance/quality control (QA/QC). Although manufacturers have greatly simplified the operation of mass spectrometers, the average mass-spectrometer laboratory will normally be staffed with several Ph.D. or M.Sc.level scientists who are considered experts in MS.

\section{Experts are a rare commodity}

The substantial growth in the use of MS has resulted in a great demand for people with expertise in this technique. The recent 50th Conference of the American Society of Mass Spectrometry, which was held in Orlando, Florida, in June 2002, had more than 100 individual employment advertisements for people skilled in the use of mass spectrometers. As in other areas of high technology, the supply of new graduates frequently falls short of the demand in industry, government and academia for these talented professionals, and academic industry and government entities are all vying for experienced people. Personnel departments in industry are finding that traditional methods to seek new talent for mass-spectrometer facilities by means of advertisements in professional magazines and journals are not productive. They are, therefore, more frequently using the services of employment agencies to fill these positions, albeit for a significant fee. However, many months are often required to fill a position, so new recruiting methods are needed. Recruiters might consider exploring new venues in the search for employees, including local MS discussion groups; the use of web sites sponsored by professional societies that attract MS professionals and that have employment sections (for example, the American Society for Mass Spectrometry, the Proteome Society and the Association of Biomolecular Resource Facilities); establishing a relationship with a university professor or department that has a recognized programme in MS; or recruiting at the major local or international MS conferences.

\section{An alternative career choice}

The current shortage of MS talent clearly opens up significant opportunities for students entering universities to study advanced degrees. Although biased after spending my entire professional career in MS, I can wholeheartedly endorse it as a profession to any new graduate student. Although the basic science of MS has been known for decades, the field constantly develops new innovations that increase its ability to analyse compounds. Some examples include bench-top GC (gas chromatography)/MS and LC/MS, new ionization methods (for example, MALDI, electrospray ionization (ESI), and atmospheric pressure photoionization (APPI) that make analysis of biomolecules routine, and new mass analysers (such as the Fourier transform ion cyclotron, quadrupole time of flight and triple quadrupole) that improve mass resolution, mass accuracy and information content. Let me provide a typical example of the influence of MS on the identification of proteins and peptides. In the mid1980s, the only viable instrument to determine the amino-acid sequence of proteins was the Edman-chemistry protein sequencer. Using Edman chemistry, one cycle of amino-acid sequence typically took $\sim 30$ minutes to perform, and required $\sim 10$ picomoles of sample. Today's mass spectrometers can identify an unknown protein using femtomoles of material in a few minutes. Much of the current proteomics research that is focused on targetprotein identification would be impossible to carry out without modern MS. However, in line with the present need throughout the drug discovery and development arena for broadly trained individuals, I would highly recommend that new students not only develop the fundamental knowledge of MS principles, but also a thorough understanding of one or more applications that are affected by its measurement capabilities.

\section{Broad knowledge is an advantage}

A new graduate that has a solid MS background is 'hot property' in today's employment environment, but this value is enhanced considerably by a strong knowledge of an application area, such as biochemistry or toxicology, that uses MS as a primary analysis tool. With a fundamental background in some aspect of the life sciences; for example, molecular or cell biology, mass spectroscopists are becoming true members of drug discovery and development teams, and are much more than just the technicians who understand the operation of mass spectrometers and can interpret the data generated by them.

John Michnowicz 